\title{
Analysis and Experiment of Compression Wave Generated by Train Entering Tunnel Entrance Hood*
}

\author{
Masanobu IIDA**, Katsuhiro KIKUCHI** and Takashi FUKUDA** $^{* *}$
}

\begin{abstract}
An analysis and a model experiment are performed on the compression wave generated by a train entering a tunnel with an entrance hood, which is a prevailing countermeasure for reducing the micro-pressure wave emitted from a Shinkansen tunnel portal. The tunnel entrance hood is a structure for extending a tunnel and has openings on its side walls or roof for decreasing the pressure gradient of the compression wave generated by train entry. The hood design has principally been performed through model tests since the three dimensionality of flow field is generally important. Thus, a more efficient design method is required. In this paper, an analytical method based on the aeroacoustic theory developed by Howe is applied to a short "acoustically compact" hood having a window on its side wall. Analytical results are in good agreement with experimental results including the effect of train offset in a double-track tunnel. It is also clarified by analysis that the performance of the entrance hood is greatly affected by the relative length of the train nose to the distance between the window and the hood entrance.
\end{abstract}

Key Words: Pressure Wave, Aerodynamic Acoustics, Three Dimensional Flow, Potential Flow, Railway, Compression Wave, Micro-Pressure Wave, Tunnel Entrance Hood, High-Speed Train

\section{Introduction}

When a train enters a tunnel at a high speed, a compression wave is generated inside the tunnel. The compression wave propagates through the tunnel at about the speed of sound and arrives at a distant portal, when an impulsive pressure wave is radiated from the portal toward the outside. The impulsive pressure wave, called the "micro-pressure wave", could cause a sudden explosive sound and/or an infrasound accompanying rattlings of window frames or shutters of nearby houses. Thus, the micro-pressure wave is one of the important impacts of high-speed railway on a wayside environment ${ }^{(1)-(5)}$.

The micro-pressure wave is generated in three stages: (1) the generation of the compression wave at the portal where the train enters, (2) the propagation of the compression wave inside the tunnel, and (3) the radiation of the micro-pressure wave from the distant tunnel portal toward the outside. In principle, it is possible to take measures

* Received 2nd March, 2006 (No. 04-0087). Japanese Original: Trans. Jpn. Soc. Mech. Eng., Vol.70, No.700, B (2004), pp.3090-3097 (Received 30th January, 2004)

** Railway Technical Research Institute, 2-8-38 Hikari-cho, Kokubunji-shi, Tokyo 185-8540, Japan against the micro-pressure wave at each of these stages, but those at stage (1), which may well be called "measures at entrance", have been extensively put into practice in Japanese Shinkansen. The "measures at entrance" reduce the pressure gradient of the wave front of the compression wave upon its generation at the tunnel entrance and are based on the following principle.

The maximum of the pressure gradient of the compression wave front can be expressed as $(\partial p / \partial t)_{\mathrm{MAX}}=$ $\Delta p / \Delta t$, where $\Delta p$ is the total pressure increase of the compression wave front and $\Delta t$ is the characteristic time for the pressure increase. The pressure gradient can be reduced by decreasing $\Delta p$ and/or increasing $\Delta t$. For inviscid flow, the total pressure increase $\Delta p$ of the compression wave is determined only by the entrance speed and the cross-sectional area ratio of the train to the tunnel

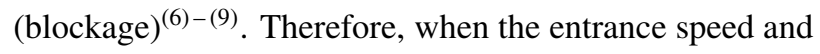
tunnel cross-sectional area are given, reducing the train cross-sectional area is the only way of decreasing $\Delta p$ (it is possible to decrease $\Delta p$ by installing an entrance hood with a length markedly larger than the overall length of the train, and thereby, the compression wave generated by nose entry can be partially counteracted by the expansion wave generated by tail entry, but such a long hood 
may be impractical in Shinkansen, where trains as long as $400 \mathrm{~m}$ run frequently). On the other hand, the characteristic time can be expressed as $\Delta t=\ell_{C} / U$ by the entrance speed $U$ and characteristic distance $\ell_{C}$, which corresponds to the distance at which the train runs while the compression wave pressure rises by $\Delta p$. When there is no hood at the portal and the train nose length is smaller than the tunnel radius $R, \ell_{C}$ is of the order $R^{(1)}$. However, $\ell_{C}$ can be increased by extending or optimizing the train nose $\mathrm{e}^{(10)-(12)}$ or by installing or extending the tunnel entrance hood ${ }^{(2),(3),(5),(13)-(17)}$. As the pressure gradient of the compression wave at the entrance is reduced by these "measures at entrance", the nonlinear steepening of the wave front of the propagating compression is relaxed, resulting in the reduction of the micro-pressure wave radiated from the distant portal. The effect of the "measures at entrance" is marked, particularly for slab-track tunnels. For example, it was reported that as much as $24 \mathrm{~dB}$ reduction in micro-pressure wave was obtained by an installation of an entrance hood at a portal of a long slab-track tunnel ${ }^{(5)}$.

In this paper, we deal with a tunnel entrance hood, a prevailing countermeasure implemented on the ground side (more than 170 portals of Shinkansen tunnels are equipped with entrance hoods $\left.{ }^{(14)}\right)$. The entrance hood is an extension of the tunnel portal having a cross-sectional area equal to or 1.5 times larger than that of the tunnel, with windows or slits placed on its side walls and/or its roof. The above discussion on the characteristic distance $\ell_{C}$ basically implies that the longer the hood, the greater the reduction in pressure gradient. Only, it is necessary to arrange suitably sized windows at suitable positions in order to utilize effectively the overall length of the hood ${ }^{(2),(3),(5),(14)-(17)}$. Designers of an entrance hood should estimate the effect of the hood length and those of the sizing and arrangement of the windows on the reduction ratio of the pressure gradient, which varies according to various conditions of the train and portal.

Figure 1 shows a photograph of a typical entrance hood at a Shinkansen tunnel portal. There are windows at both side walls of the hood. Because Shinkansen tunnels are double-tracked, the train runs with an offset in the

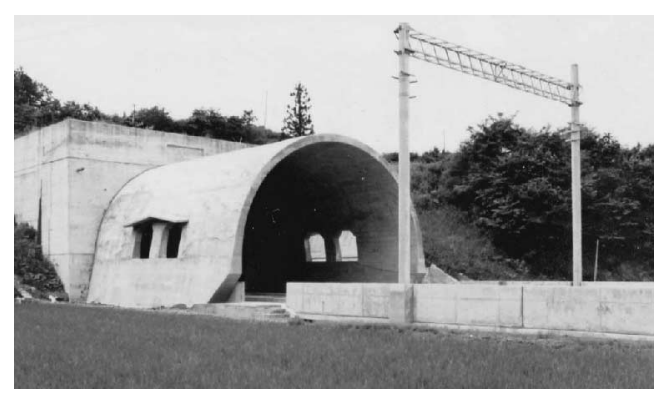

Fig. 1 Tunnel entrance hood in Shinkansen horizontal direction from the center axis of the tunnel and hood toward either of the side walls. Consequently, three dimensionality in geometry has a large effect on the waveform of the compression wave and its gradient. This was confirmed by the results of field tests (p.74 in Ref. (2)) and a model experiment ${ }^{(16)}$. Thus, when performing a numerical simulation (CFD) on the entrance hood, it is necessary to calculate the three dimensional unsteady flow. However, considering that even a high-performance computer consumes a huge amount of CPU time for 3D CFD on structure with a complex geometry, such as an entrance hood, we cannot say that CFD is necessarily suitable for the design process, which requires repeated calculations for many hood shape candidates. Compared with CFD, model-scale testing has a large data productivity. For example, the authors' apparatus allows 3 to 5 runs of tunnel entry experiment in an hour. Hence, the authors have estimated the performance of the entrance hood from the data obtained by the model test.

Recently, an analytical method based on an acoustic theory has been developed by Howe $\mathrm{e}^{(9),(18),(19)}$ to predict rapidly the compression wave generated by a train entering a tunnel. Analyses have been performed on a circular cylindrical tunnel, a porous hood and a flared portal. The validity of these analyses was confirmed for an optimally flared portal by comparison with the authors' experiment $^{(19)}$. Furthermore, concerning the case where a train advances along the center axis of the tunnel having a rectangular window on its wall, an analysis and an experiment were performed on the relationship between the compression wave and the jet flow from the window ${ }^{(20)}$. Incidentally, an analytical method that considers the steady flow around the train has been developed to predict semiempirically the compression wave by a train entering a tunnel ${ }^{(21)}$, but to the authors' knowledge, this method has not been applied to a tunnel portal with an entrance hood having a window on its wall.

There are two objectives in this study. The first objective is to apply the analytical method developed by Howe to the design of entrance hoods. In this paper, we treat only a simple short hood with the same circular cross section as the tunnel, having a rectangular window on its wall, but the train not only advances along the tunnel axis but also is offset from it, which enables us to investigate the effect of geometrical three dimensionality. We also perform an experiment in order to validate the analysis. The second objective is to obtain a guiding principle for the design of entrance hoods. In particular, we note the connection of an optimum position of a window with the nose length of the entering train. To this end, parametric analyses are performed for different train nose lengths. 


\section{Analytical Method}

\subsection{Analytical model}

Figure 2 shows an analytical model. To pay attention to the entrance of the nose, both the tunnel and train are assumed to have semi-infinite lengths. The origin is located at the center of the entrance plane of the hood with a circular cross section (we regard the part of the cylindrical tunnel from the entrance to the center of the window as the hood). The $x$-axis is along the hood and tunnel axis (the positive direction is from the tunnel to the outside free space), the $y$-axis in the vertical direction, and the $z$ axis in the horizontal direction. To simulate a double-track tunnel, the center axis of the train is offset from the hoodtunnel axis in the $z$-direction by the distance $z_{T}$. The cross section of the hood is the same as that of the tunnel, that is, a circle of radius $R$ and area $A=\pi R^{2}$. There is a rectangular window of axial length $\ell_{w}=0.8 R$ and azimuthal length $h_{w}=0.4 R$ at the position of $x=-1.5 R$. The model shown in Fig. 2 is a simplified version of a short hood in a $10 \mathrm{~m}$ range (the length of entrance hoods in Shinkansen ranges from 10 to $49 \mathrm{~m}$, and the number of entrance hoods in $10 \mathrm{~m}$ range is the largest among all the hoods). The length of the train nose is $\ell_{n}$ and the cross section of the train is a circle of radius $h$ and area $A_{0}=\pi h^{2}$. Accordingly, the cross-sectional area ratio of the train to the tunnel is $A_{0} / A=h^{2} / R^{2}$. The model shown in Fig. 2, where the cross sections of the train and tunnel are represented as circles, is based on the concept of the mirror image expression of a ground plane effect ${ }^{(19),(22)}$.

\subsection{Analysis of compression wave}

Hereafter, an outline of the analytical method is given (see Ref. (19) for details). The main cause of the compression wave generation by a train entering a tunnel is the effect of air displacement by the advancing train nose
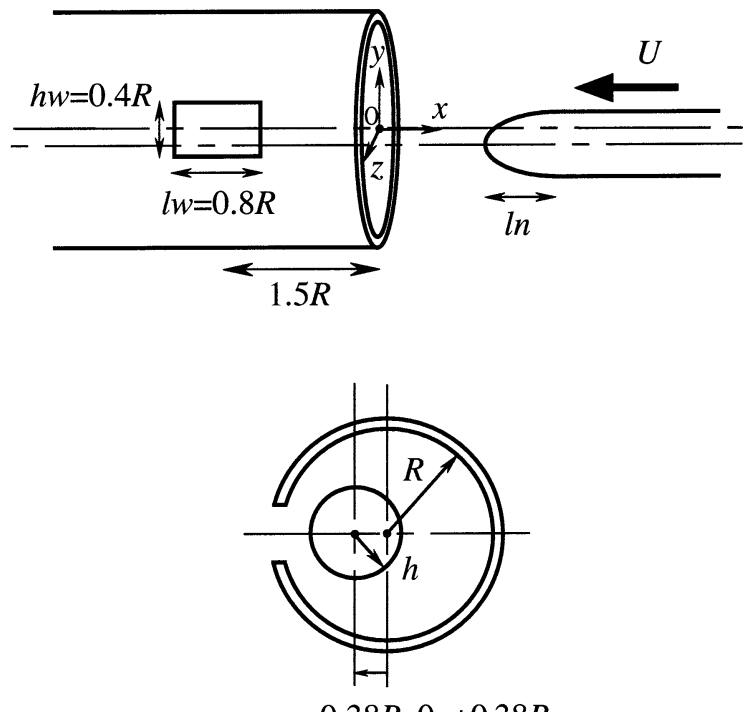

$z T=-0.38 R, 0,+0.38 R$

Fig. 2 Analytical model (piston effect). From the viewpoint of acoustics, the train nose is a monopole source. Consequently, the tunnel entry problem can be formulated as an acoustic problem of sound generation by the interaction of a moving monopole source and the tunnel portal ${ }^{(8),(9)}$.

Since the train entrance Mach number $M$ is much less than 1 , the compression wave propagates through the tunnel as a plane wave whose dominating frequency is below the cutoff frequency of the tunnel duct. Therefore, the pressure of the compression wave at the position $x$ in the tunnel and the time $t(t=0$ when the train nose enters the entrance) is expressed as $p(x, t)$ when the observer is in the far field within the tunnel (but not as far as the nonlinear steepening of the compression wave becomes important). In high-speed railways such as Shinkansen, the cross-sectional area ratio of the train to the tunnel is sufficiently smaller than 1 and the train nose is streamlined. Hence, we can adopt the slender body approximation and substitute the sources distributed originally on the train surface with those distributed along the train axis. Moreover, we neglect the contributions from the vorticity in shear layers of jets formed at the hood entrance and the rectangular window, and those from viscous friction at the surface of the train and the tunnel wall. From these assumptions and approximations, the governing equation of the compression wave generation is given as the inhomogeneous wave equation of

$$
\left(\frac{1}{c_{0}^{2}} \frac{\partial^{2}}{\partial t^{2}}-\nabla^{2}\right) p=\rho_{0} U \frac{\partial}{\partial t}\left(\frac{\partial A_{T}}{\partial x}(x+U t) \delta(y) \delta\left(z-z_{T}\right)\right),
$$

where $\rho_{0}$ : the density, $c_{0}$ : the sound speed, $U$ : the entrance speed, $A_{T}(x)$ : the cross-sectional area distribution of the train nose, and $\delta(x)$ : the Dirac delta function.

In this paper, we treat a short hood whose length (the distance between the entrance and the center of the window) is $1.5 R$, which is smaller than the tunnel diameter. Hence, the wavelength of the compression wave is sufficiently larger than the representative length of the hood, and therefore, the hood can be regarded as acoustically compact. The adequacy of this assumption is confirmed by comparing analytical results with experimental results, as shown in section 3.2. When the hood is longer, for example $10 R$ in length, and cannot be regarded as compact in the $x$-direction, though still compact in the $y$ - and $z$ directions, we should perform an analysis by considering the difference between wave phases at different positions of $x$ in the hood ${ }^{(20)}$. However, when the compact approximation holds as in the present case, Green's function of the wave equation can be simplified, and we can solve Eq. (1) in the form of convolution integral as

$$
p_{1}(x, t)=\frac{\rho_{0} U^{2}}{A\left(1-M^{2}\right)} \int_{-\infty}^{\infty} \frac{\partial A_{T}}{\partial x^{\prime}}\left(x^{\prime}+U[t]\right) \frac{\partial \phi^{*}}{\partial x^{\prime}}\left(x^{\prime}, 0, z_{T}\right) d x^{\prime},
$$


where $[t]=t-|x| / c_{0}$ : the retarded time, $x^{\prime}$ : the coordinate of the direction from the nose to the tail, and $\phi^{*}(x, y, z)$ : the velocity potential of an incompressible flow determined solely by the hood-tunnel entrance geometry. (The reason why the pressure is written as $p_{1}(x, t)$ is explained below.) The coefficient $1 /\left(1-M^{2}\right)$ before the integral on the right-hand side is for the correction of Mach number dependency, which extends the validity of the solution up to $M \sim 0.4^{(18)}$.

When the train enters the tunnel, the pressure ahead of the train in the tunnel rises and the aerodynamic drag acting on the train nose increases. Acoustically, the reaction of the increased drag, which is exerted on the air, is equivalent to a dipole source. The pressure in Eq. (2) is a component of a monopole source and therefore written as $p_{1}(x, t)$. On the other hand, the pressure component of the dipole source, which is written as $p_{2}(x, t)$, is given as ${ }^{(19)}$

$$
p_{2}(x, t)=\frac{p_{1}(x, t)}{A} \int_{-\infty}^{\infty} \frac{\partial A_{T}}{\partial x^{\prime}}\left(x^{\prime}+U[t]\right) \frac{\partial \phi^{*}}{\partial x^{\prime}}\left(x^{\prime}, 0, z_{T}\right) d x^{\prime}
$$

This result is obtained by estimating the strength of the dipole sources distributed on the train nose surface, using Bernoulli's equation described in the coordinate system fixed on the train ${ }^{(19)}$. (The strength of the monopole source is determined by the train nose geometry only and therefore does not vary with time, but that of the dipole source varies with time because the drag acting on the nose increases as the nose enters the tunnel. The change of the dipole source is estimated by quasi-static approximation, with the application of the steady Bernoulli's equation at each instant. This is because the contribution from the unsteady term of the equation of motion is smaller than that from the convection term, in the coordinate system fixed on the train. The Strouhal number St, which indicates the ratio of the two terms, is roughly estimated as $S t \sim 0.5 \sqrt{A_{0} / A}$, since the main frequency of the flow change by train entry is of the order $U / 2 R$ and the train radius $h=R \sqrt{A_{0} / A}$. In general, the cross-sectional area ratio of the train to the tunnel is much smaller than 1 in high-speed railways, and therefore, the Strouhal number is also small.)

From Eq. (3), we observe that $p_{2}$ is smaller than $p_{1}$ by the order of the cross-sectional area ratio of the train to the tunnel $A_{0} / A$, because $\partial \phi^{*} / \partial x^{\prime} \sim O(1)$. The crosssectional area ratio of the train to the tunnel $A_{0} / A$ ranges from 0.12 to 0.22 for most high-speed railways, and then if we neglect the component $p_{2}$, an error more than $10 \%$ will be produced. Accordingly, we consider the dipole component and express the pressure of the compression wave $p(x, t)$ as the sum of $p_{1}$ and $p_{2}$

$$
p(x, t)=p_{1}(x, t)+p_{2}(x, t) .
$$

The pressure gradient $\partial p / \partial t$, which is important in the estimation of the micro-pressure wave, is determined by dif- ferentiating Eqs. (2) - (4) with $t$ and integrating by parts, as

$$
\begin{aligned}
\frac{\partial p_{1}}{\partial t} & =-\frac{\rho_{0} U^{3}}{A\left(1-M^{2}\right)} \int_{-\infty}^{\infty} \frac{\partial A_{T}}{\partial x^{\prime}}\left(x^{\prime}+U[t]\right) \frac{\partial^{2} \phi^{*}}{\partial x^{\prime 2}}\left(x^{\prime}, 0, z_{T}\right) d x^{\prime} \\
\frac{\partial p_{2}}{\partial t} & =\frac{2}{A} \frac{\partial p_{1}}{\partial t} \int_{-\infty}^{\infty} \frac{\partial A_{T}}{\partial x^{\prime}}\left(x^{\prime}+U[t]\right) \frac{\partial \phi^{*}}{\partial x^{\prime}}\left(x^{\prime}, 0, z_{T}\right) d x^{\prime} \\
\frac{\partial p}{\partial t} & =\frac{\partial p_{1}}{\partial t}+\frac{\partial p_{2}}{\partial t}
\end{aligned}
$$

The principal waveform of the pressure gradient is determined by $\partial p_{1} / \partial t$. The function $\partial^{2} \phi^{*} / \partial x^{\prime 2}$ in the integrand on the right-hand side of the equation for $\partial p_{1} / \partial t$ represents the geometric inhomogeneity around the hood and exhibits abrupt variations in the vicinity of the edges of the hood entrance and the window, as shown in section 2.3. The above equation shows that in a geometrically homogeneous space, such as a free space or inside a uniform tunnel, where $\partial^{2} \phi^{*} / \partial x^{\prime 2}=0$, no pressure waves are generated. However, when sources travel in a geometrically inhomogeneous space, such as, the tunnel portal or the hood, pressure waves are generated even if the source strength does not change.

\subsection{Calculation of potential function}

The function $\phi^{*}(x, y, z)$, which is introduced in section 2.2 , is the velocity potential of an incompressible flow out of the tunnel in the presence of a piston moving at a unit speed in a positive $x$-direction at a large distance from the entrance within the tunnel. As to a thin-walled circular cylindrical tunnel with an unflanged portal, an exact solution of $\phi^{*}$ is known ${ }^{(9)}$. Furthermore, when a window exists at a large distance from the hood entrance, analytical treatment is also possible using the model of a window on the wall of a tunnel extending infinitely in both directions ${ }^{(20)}$. However, in the present case where a window exists near the entrance, $\phi^{*}$ must be determined numerically. In this study, we calculate $\phi^{*}$ by the three-dimensional boundary element method (BEM) based on the Laplace equation. The BEM program has been used for the study of pressure variations due to two trains passing each other ${ }^{(23)}$. Figure 3 shows boundary elements for the present hoodtunnel model. The overall length of the hood and tunnel is $10 R$, and the calculation is performed by setting a piston

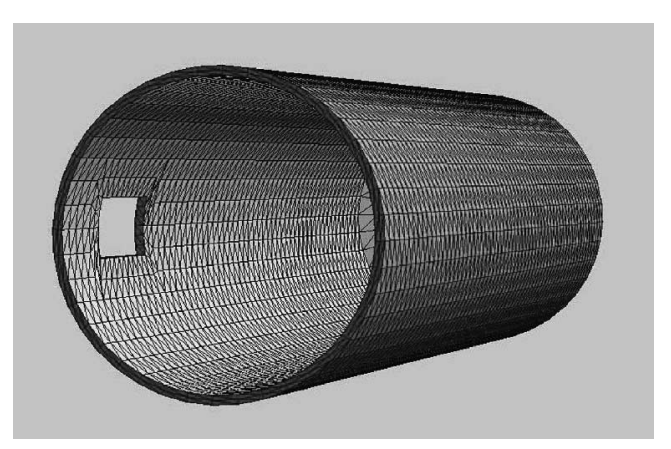

Fig. 3 Boundary elements 

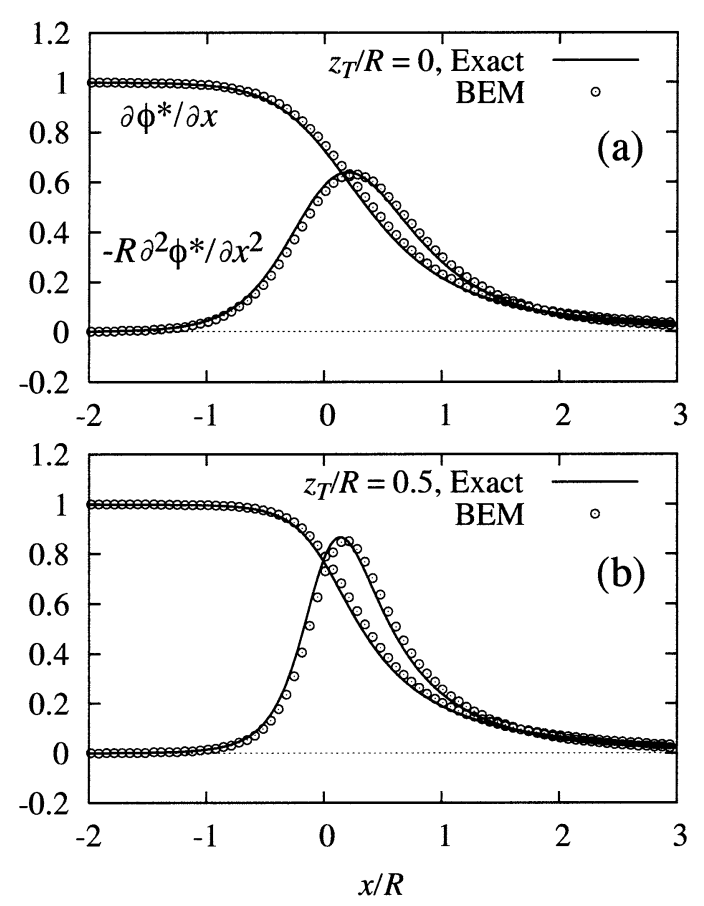

Fig. 4 Exact and BEM solutions of $\phi^{*}$; no window, $z_{T} / R=0$ and 0.5

surface moving at a unit speed at the inner end of the tunnel. The motion of the piston is treated in the same manner as that of a passing train ${ }^{(23)}$. The division number of the boundary elements is determined through repeated calculations with the division number being increased until the calculated value of $\partial^{2} \phi^{*} / \partial x^{\prime 2}$ converges. The number of boundary elements for the hood model in Fig. 3 is 5184 .

To validate the BEM, we compare the numerical result with the exact result ${ }^{(9)}$ for the unflanged, thin-walled circular cylindrical tunnel. Figure 4 shows the results when the train and tunnel axes coincide with each other $\left(z_{T}=0\right)$ or not $\left(z_{T}=0.5 R\right)$. From the figure, we can confirm that the present BEM is sufficiently accurate.

Figure 5 shows numerical results for the effects of the window and the offset of the train axis on $\phi^{*}\left(x, 0, z_{T}\right)$. Without the window, $-R \partial^{2} \phi^{*} / \partial x^{\prime 2}$ has an impulsive form having only one peak, while with the window, two peaks appear. This indicates, as stated earlier, that the source (train nose) interacts with two parts of the hood (the entrance and window) and generates pressure waves twice. We can also observe, irrespective of the existence of the window, that when the train axis does not coincide with the tunnel axis and is located near the edge of the entrance or the window, the peak heights of $-R \partial^{2} \phi^{*} / \partial x^{2}$ increase, and therefore, stronger interactions occur.

From Fig. $5(\mathrm{~b})$, the two peaks of $-R \partial^{2} \phi^{*} / \partial x^{\prime 2}$ have an approximately equal height when $z_{T}=+0.38 R$, or the train axis is displaced toward the window (near-window position). In the limit as the train nose is shortened $\left(\ell_{n} \rightarrow 0\right), \frac{1}{A_{0}} \frac{\partial A_{T}}{\partial x}(x) \rightarrow \delta(x)$. Hence, from Eq. (5), the cor-
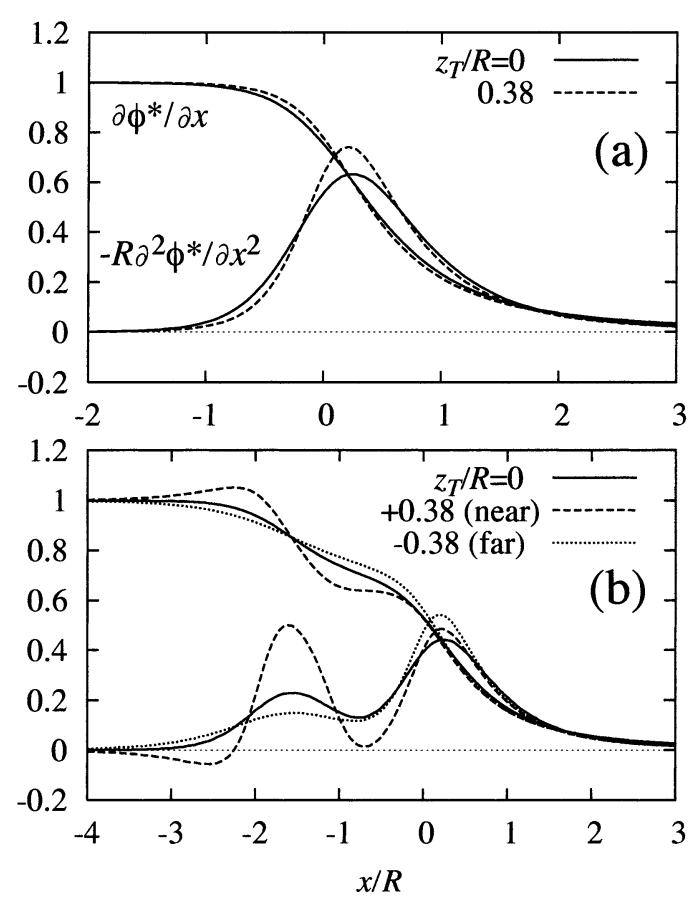

Fig. 5 BEM solutions of $\phi^{*}$; (a) no window, $z_{T} / R=0$ and 0.38 , and (b) with a window, $z_{T} / R=0$ and \pm 0.38

responding two peaks of the pressure gradient generated by the monopole source $\partial p_{1} / \partial t$ are also equal in height under this offset condition. If we neglect the contribution of the dipole source, which is smaller than that of the monopole source by the order of the cross-sectional area ratio of the train to the tunnel, we can say that the two peaks of the total pressure gradient $\partial p / \partial t$ of the compression wave also have nearly the same height. This indicates that the size (or area) of the window is optimized under the offset condition of $z_{T}=+0.38 R$. On the other hand, when $z_{T}=0$, or the train axis coincides with the tunnel axis, and when $z_{T}=-0.38 R$, or it is displaced away from the window (far-window position), the two peaks of $-R \partial^{2} \phi^{*} / \partial x^{2}$ are not equal in height, that is, the first peak at $x / R \sim 0.1$ is larger than the second peak at $x / R \sim-1.7$. Considering the properties of the potential flow $\phi^{*}$, the larger the window, the higher the second peak. Consequently, we can predict that by increasing the window area from that shown in Fig. 2, the two peaks will be made equal, and thus, the hood performance will be optimized in the cases of $z_{T}=0$ and $-0.38 R$. In this manner, we can obtain useful knowledge for optimizing the window only from information on the hood geometry without actually performing the unsteady flow calculation of the tunnel entry problem. This will be a great advantage of the method. (When the train nose is longer, these remarks do not necessarily hold, as discussed in section 4.)

\section{Comparison between Analysis and Experiment}

\subsection{Experimental apparatus}

A model experiment was performed in order to vali- 


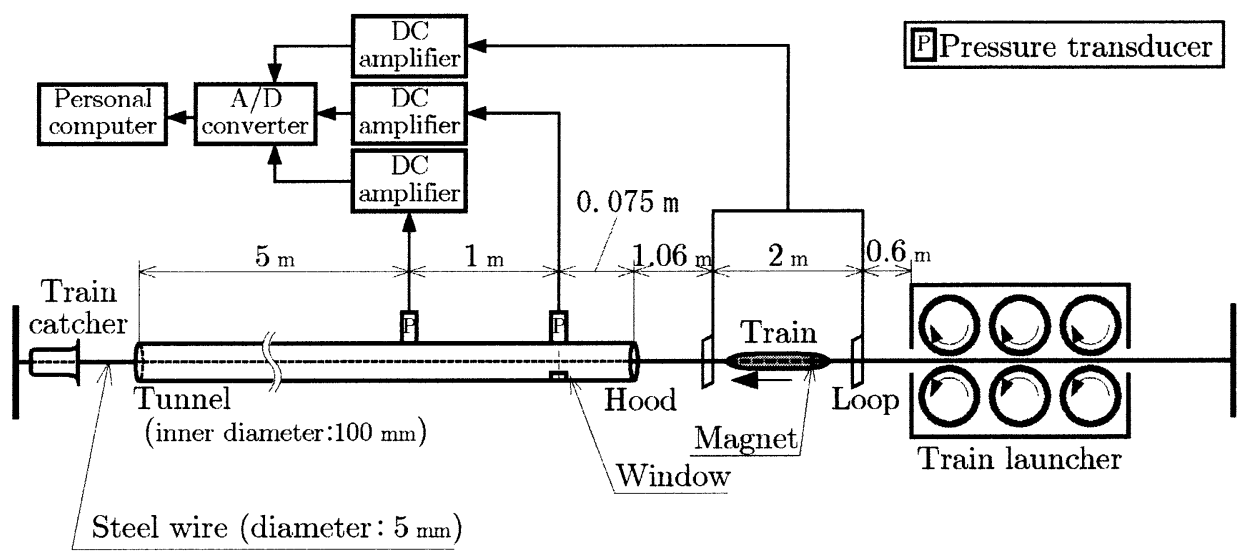

Fig. 6 Experimental apparatus

date the analytical results for the compression wave. The apparatus projects an axisymmetric model train, made of plastics and guided along a stretched steel wire, into a circular cylindrical tunnel, made of vinyl chloride, by a launcher consisting of three pairs of rotating wheels (Fig. 6). A rectangular window exists on the wall of the model tunnel $(R=50 \mathrm{~mm})$, as in the analytical model shown in Fig. 2. The model tunnel was built such that its window can be closed if necessary. Pressure sensors (Toyoda Machine Works PD104K) are installed on the inner surface of the tunnel wall. When the launching of the model train is detected, pressure signals are stored on a personal computer, after being amplified by a Toyoda Machine Works AA6210 amplifier and digitized by an A/D converter. The model train used in the experiment has the cross-sectional area ratio of the train to the tunnel $A_{0} / A=0.2$. The nose part has an axisymmetric shape of a half ellipsoid and a length of $\ell_{n}=1.34 R$. The offset condition is set by displacing the model tunnel in the horizontal direction from the stretched wire that coincides with the train axis and is fixed to the laboratory floor. Throughout the experiment, the launching speed, which is practically equal to the tunnel entrance speed, is set to be $U=300 \mathrm{~km} / \mathrm{h}(M=0.245)$. The entrance speed is measured by detecting the magnetic field generated by permanent magnets embedded in the train as the train passes through two wire loops placed $2 \mathrm{~m}$ apart in front of the entrance. The error of the speed measurement is estimated to be no more than $\pm 1 \%{ }^{(19)}$.

\subsection{Results of comparison}

First, we observe the results of comparison for the case without the window. Figure 7 shows the compression wave generated when $z_{T} / R=0$ and 0.38 . Hereafter (including Fig. 7), the pressure and its gradient are nondimensionalized as $p / \Delta p_{t}$ and $(\partial p / \partial t) /\left(U \Delta p_{t} / R\right)$, respectively, with the reference pressure given theoretically ${ }^{(19)}$

$$
\Delta p_{t}=\frac{\rho_{0} U^{2}}{\left(1-M^{2}\right)} \frac{A_{0}}{A}\left(1+\frac{A_{0}}{A}\right)
$$

and the reference time $R / U$. The horizontal axis of the fig-
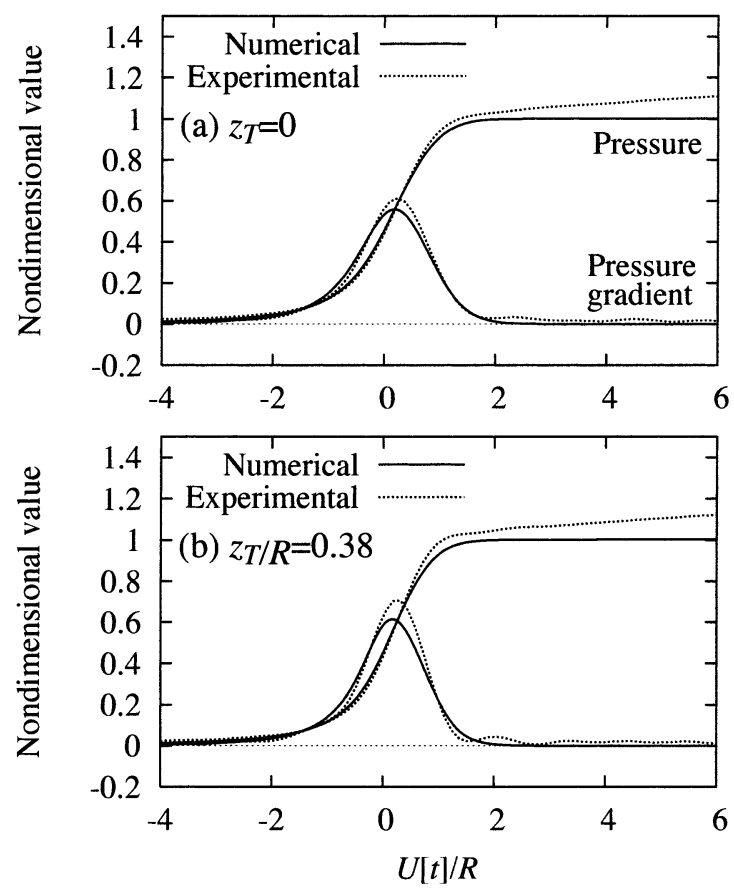

Fig. 7 Numerical and experimental results for compression wave; no windows, $z_{T} / R=0$ and 0.38

ure represents the nondimensional retarded time $U[t] / R$. The analytical and experimental results for the compression wave are in good agreement under both offset conditions. In the experiment, the pressure continues to rise gradually after the principal wave front is formed at about $U[t] / R=2$, which is not observed in the analytical result. This is mainly because viscous friction on the surfaces of the train and tunnel wall ${ }^{(24)}$ is neglected in the analysis. As to the pressure gradient, we observe that the analysis shows an approximately $5 \%$ smaller peak than the experiment. One of the reasons is considered to be the effect of the slender body approximation, i.e., the line source distribution. This effect becomes greater as the cross-sectional area of the train to the tunnel becomes larger. The value of $A_{0} / A=0.2$ is far from being large and yet is slightly larger 

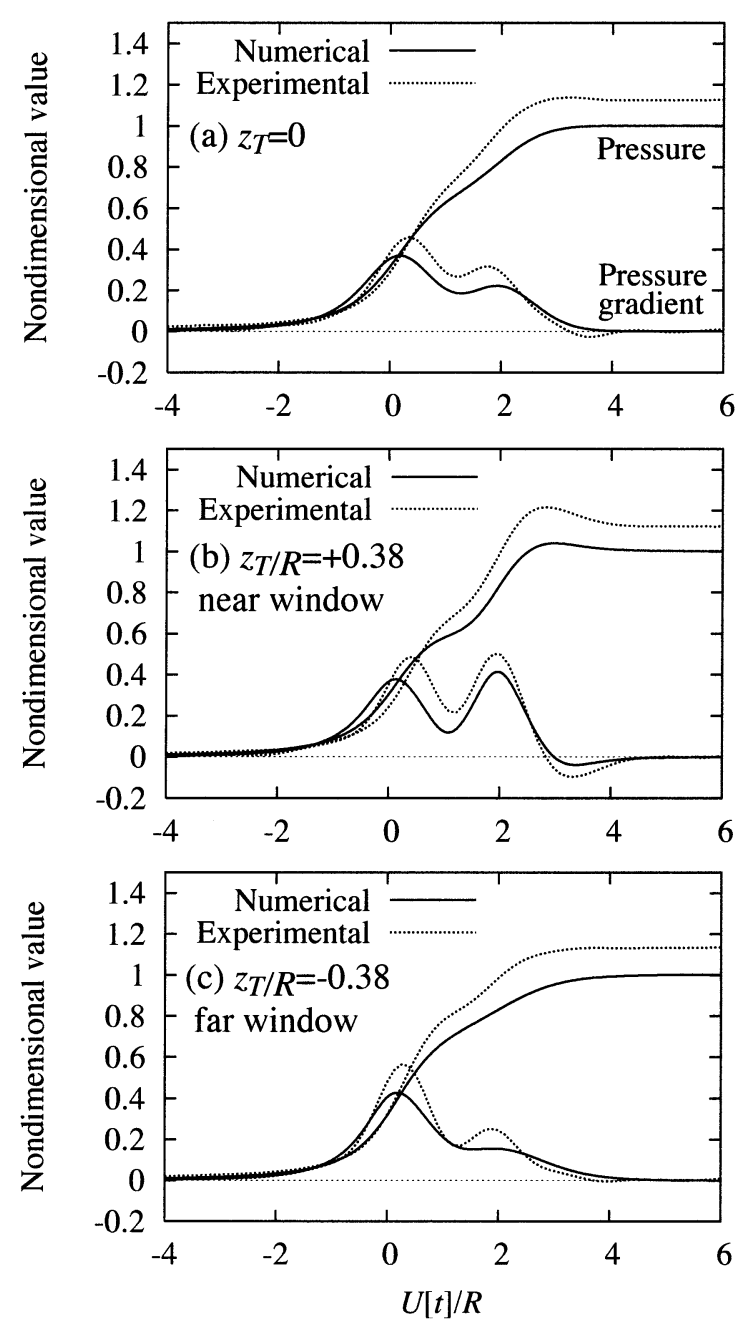

Fig. 8 Numerical and experimental results for compression wave; with a window, $z_{T} / R=0$ and \pm 0.38

than those of most recent Shinkansen trains. In the line source model, the sources are distributed along the train axis line, while they are actually distributed on the train surface, and the train travels along the line farther from the edges of the entrance and the window than in the surface source model. As a result, the pressure gradient calculated using the line source model is smaller. This tendency becomes more marked as the ratio of the train radius to the tunnel radius, or the cross-sectional area ratio, becomes larger.

Second, we observe the results of comparison for the case with the window. Figure 8 shows the compression wave when $z_{T} / R=0$ and \pm 0.38 . According to the definition of the coordinate system, $z_{T}>0$ is the case when the model train travels along the line near the window. Similar to the case without the window, the analytical and experimental results for the compression wave are in good agreement under all offset conditions. All the principal characteristics of the waveform are commonly observed: (1) two peaks exist in the pressure gradient in the presence of a window, (2) the smallest peak value is obtained when the train travels along the tunnel axis line, (3) the peak corresponding to the passing by the window is larger when the train travels along the line near the window, and (4) the peak corresponding to the passing by the entrance is larger when the train travels along the line far from the window. These effects of the train axis offset cannot be reproduced in the analyses or simulations based on an axisymmetric flow model unless some special treatment on three dimensionality is considered. By observing the pressure gradient waveform shown in Fig. 8, the timings of the two peaks are considered in agreement between the analysis and the experiment. In other words, the analysis gives correct results for the phase of the compression wave, confirming the validity of the compact assumption for the present short hood.

The following can be pointed out when we observe the comparison results in more detail. The compression wave pressure of the experiment is greater than that of the analysis after the train nose enters the tunnel $(U[t] / R \sim 6)$; the reason for this is, as in the case without the window, that the viscous friction on the surfaces of the train and tunnel wall is neglected in the analysis. However, as to the result that the pressure is underestimated in the analysis at about $U[t] / R=2.5$, when the compression wave pressure attains its maximum, we should consider other reasons because the effect of the viscous friction on the train surface is not marked around these times. Concerning this, a detailed analysis and an experiment were performed separately, and the following results were obtained ${ }^{(20)}$. In this paper, we assume a spherically spreading, irrotational source flow out of the window. However, in reality, during the time interval from the nose entry into the entrance until the nose passing by the window, the flow out of the window separates at the window edges and forms an unsteady jet. According to a theorerical analysis ${ }^{(20)}$, vorticity in the shear layer of the jet produces a positive pressure pulse within the hood. This implies that if we consider this additional pressure due to the jet, the compression wave pressure shown in Fig. 8 will be larger. Next, we also observe in the figure that the pressure gradient of the experiment is also greater than that of the analysis on the whole. One reason may be the above-mentioned effect of the line source approximation. However, a more acceptable reason may be the effect of the jet out of the window. The effect of the jet appears during the time from the arrival at the window of the compression wave generated by train entry into the entrance until the passing by the window of the nose. Therefore, the longer the distance between the hood entrance and the window (the longer the hood), the greater the effect of the jet on the compression wave.

\section{Analysis on Relationship between Nose Length and Window Position}

The present analysis has been validated in section 3 


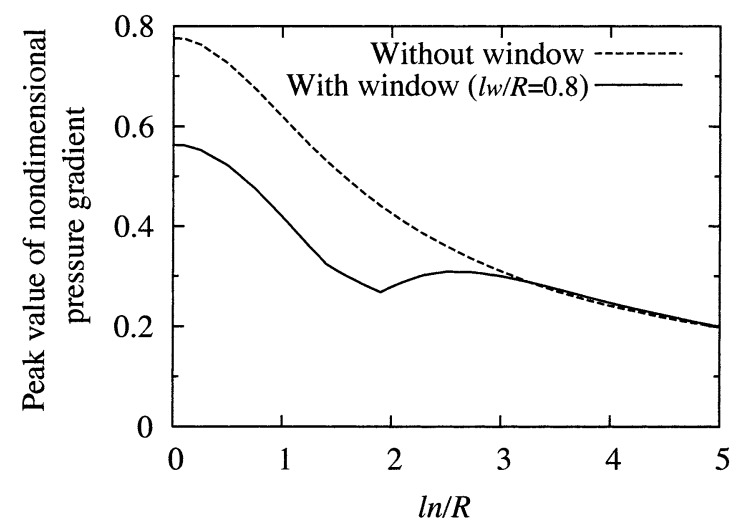

Fig. 9 Relationship between train nose length and peak value of pressure gradient

by comparison with the experiment for the compression wave generated not only in a simple circular cylindrical tunnel but also in a hood with a rectangular window on its wall. We now determine, to demonstrate the applicability of the method, how the hood effect of reducing the pressure gradient varies with the train nose length.

The analytical hood model is the same as that in sections 2 and 3 (Fig. 2). The train nose length ranges from $\ell_{n} / R=0$ to 5 , and the cross-sectional area of the nose linearly changes with the distance from the nose tip (the corresponding axisymmetric body is a paraboloid of revolution). The offset of the train axis is $z_{T} / R=+0.38$, and therefore, the train travels along the line near the window. As mentioned in section 2.3, the size of the window is nearly optimized under this offset condition.

For the two cases with and without the window, we repeat the calculation of the compression wave, changing the length of the train nose gradually. Figure 9 shows the relationship between the nose length and the maximum peak value of the pressure gradient, obtained by this parametric calculation. Without the window, the maximum peak value of the pressure gradient decreases monotonically as the nose length increases. On the other hand, with the window, the maximum peak value of the pressure gradient decreases as the nose length increases from $\ell_{n} / R=0$ to 1.9 , increases as the nose length increases from $\ell_{n} / R=1.9$ to 2.5 , and decreases again as the nose length increases from $\ell_{n} / R=2.5$ up. When $\ell_{n} / R<3.2$, the maximum peak value of the pressure gradient is smaller in the case with the window than in the case without the window, for the same nose length. However, when $\ell_{n} / R>3.2$, the peak values of the cases with and without the window are similar. In other words, the reducing effect of the pressure gradient by the window vanishes when $\ell_{n} / R>3.2$.

Figure 10 depicts compression waves and their pressure gradients for the train nose lengths $\ell_{n} / R=1,1.5,2$ and 2.5. From this, we can observe two peaks of the pressure gradient when $\ell_{n} / R=1$ and 1.5 , and another peak, the third peak, which is larger than the other two peaks, when
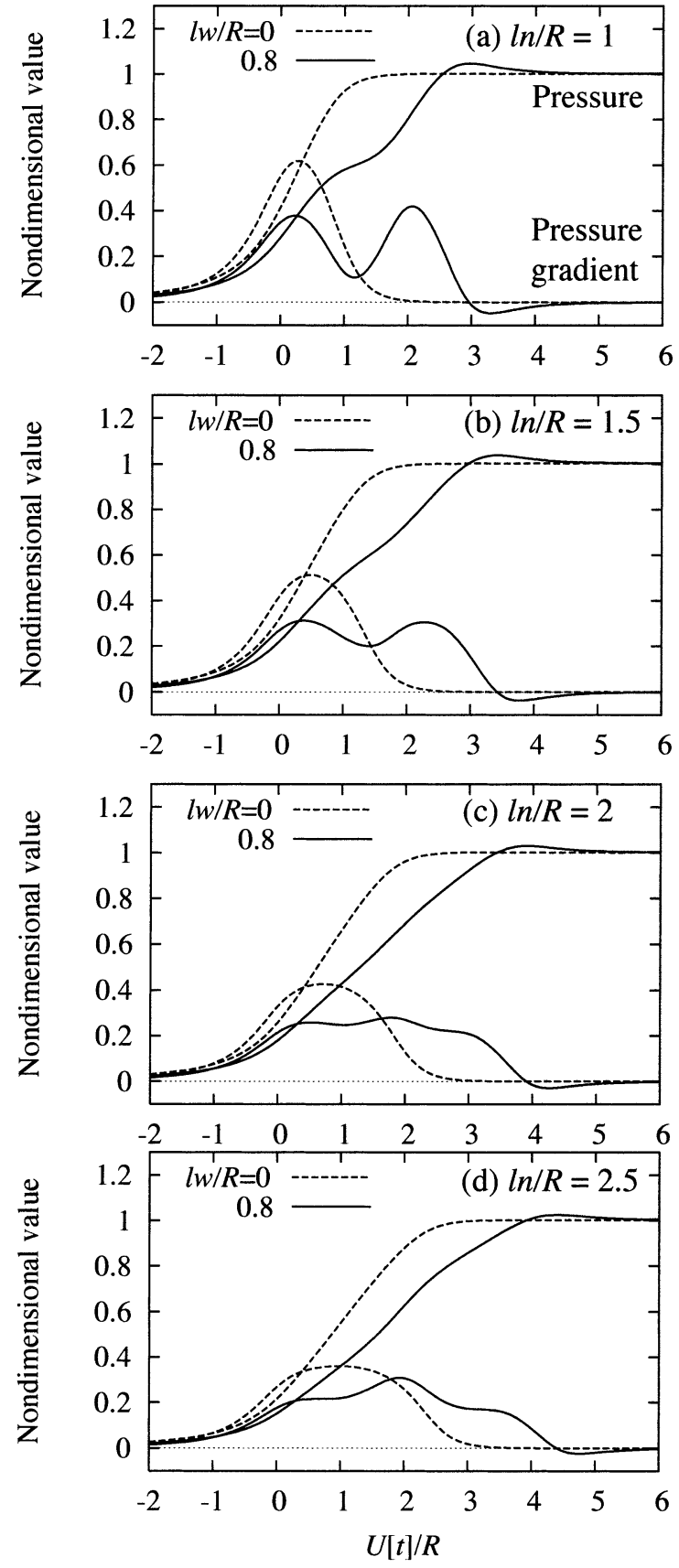

Fig. 10 Compression waves for train nose lengths $\ell_{n} / R=1,1.5$, 2 and $2.5 ; z_{T} / R=+0.38$

$\ell_{n} / R=2$ and 2.5. Without the window, the compression wave is generated only once by the interaction between the nose and the entrance, and the longer the nose, the larger the time width of the wave front. On the other hand, with the window, the compression wave is generated twice by the interaction between the nose and the entrance and between the nose and the window. The time width of each compression wave front increases with the nose length, and when the nose length is larger than about $1.9 R$ (the same order of the distance between the entrance and the center of the window of $1.5 R$ ), the two compression waves 
considerably overlap with each other and an apparent third peak emerges between the other two peaks. This overlapping mechanism explains the distinctive result shown in Fig. 9, that is, the pressure gradient does not necessarily decrease as the nose length increases in the case with the window. Furthermore, the results similar to those shown in Fig. 10 are also obtained in model experiments. Figure 8 (b) in section 3 shows the experimental result for the pressure gradient when $\ell_{n} / R=1.34$, though the nose shape of the ellipsoid is different. There are two peaks in Fig. 8 (b), which corresponds to Fig. 10 (b). In addition, the pressure gradient waveform whose middle part juts out as in Fig. 10 (c) and (d) agrees qualitatively with the result of another experiment ${ }^{(15)}$ using models of a long nose train and a short hood.

These observations lead us to a practically important guidance that the distance between the entrance and the window center should be equal or longer than the order of the nose length. Moreover, if we stretch this guidance, in the case of an entrance hood with multiple windows, the distances between neighboring windows should also be equal or longer than the order of the nose length. However, entrance hoods with multiple windows usually have large overall lengths, and compactness in the $x$-direction assumed in the present analysis may not be satisfied. Therefore, long hoods should be investigated further.

\section{Conclusions}

An analysis and a model experiment were performed on the compression wave generated by a train entering a tunnel with an entrance hood, which is sufficiently short to be regarded as acoustically compact, has the same circular cross section as the tunnel and has a rectangular window on its side wall. The analytical method used is based on the aeroacoustic theory developed by Howe, taking advantage of the boundary element method (BEM) for calculating the potential flow through the portal of arbitrary geometry. The results of the analysis and experiment including the effect of the train axis offset were in good agreement. Thus, the validity of the analytical method was confirmed for three dimensional problems, such as those in portals with hoods. The method could supply the compression waveform in a short calculation and therefore could be a useful tool for hood design. Furthermore, it was clarified by analysis that the performance of the hood is greatly affected by the relative length of the train nose to the distance between the window and the entrance. Moreover, a practically important guidance was obtained, that is, the distance between the entrance and the window center should be equal or longer than the order of the nose length.

In this paper, we dealt with a short "acoustically compact" hood. For long hoods that are not necessarily compact in the direction of the tunnel axis, further research is necessary.

\section{References}

(1) Yamamoto, A., Micro-Pressure Wave from Tunnel Exit, Preprint of the Physical Society of Japan, (in Japanese), (1977).

(2) Ozawa, S., Studies of Micro-Pressure Wave Radiated from a Tunnel Exit, Railway Technical Research Report, (in Japanese), No.1121 (1979).

( 3 ) Ozawa, S., Maeda, T., Matsumura, T., Uchida, K., Kajiyama, H. and Tanemoto, K., Countermeasures to Reduce Micro-Pressure Waves Radiating from Exits of Shinkansen Tunnels, Proc. 7th Int. Symp. Aerodynamics and Ventilation of Vehicle Tunnels, (1991), pp.253266.

( 4 ) Matsuo, K., Wave Phenomena in High-Speed Railway, Science of Machine, (in Japanese), Vol.45, No.6 (1993), pp.38-43.

( 5 ) Maeda, T., Aerodynamic Characteristics of Train and Countermeasures for Decreasing Micro-Pressure Wave, RTRI Report, (in Japanese), Special No.20 (1998).

( 6 ) Hara, T., Aerodynamic Force Acting on a High-Speed Train at Tunnel Entrance, Bulletin of JSME, Vol.4, No.15 (1961), pp.547-553.

(7) Yamamoto, A., Aerodynamics of Train and Tunnel, Railway Technical Research Report, (in Japanese), No.1230 (1983).

( 8 ) Sugimoto, N., Sound Field in a Tunnel Generated by Traveling of a High-Speed Train, Theoretical and Computational Acoustics, Vol.1 (1994), pp.45-56.

(9) Howe, M.S., The Compression Wave Produced by a High-Speed Train Entering a Tunnel, Proc. R. Soc. Lond., A, Vol.454 (1998), pp.1523-1534.

(10) Maeda, T., Matsumura, T., Iida, M., Nakatani, K. and Uchida, K., Effect of Shape of Train Nose on Compression Wave Generated by Train Entering Tunnel, Proc. Int. Conf. Speedup Tech. Rail. Maglev Vehicles, Vol.2 (1993), pp.315-319.

(11) Iida, M., Matsumura, T., Fukuda, T., Nakatani, K. and Maeda, T., Optimization of Train Nose Shape for Reducing Impulsive Pressure Wave from Tunnel Exit, Trans. Jpn. Soc. Mech. Eng., (in Japanese), Vol.62, No.596, B (1996), pp.1428-1435.

(12) Ogawa, T. and Fujii, K., Theoretical Algorithm to Design a Train Shape for Alleviating the Booming Noise at a Tunnel Exit, Trans. Jpn. Soc. Mech. Eng., (in Japanese), Vol.62, No.599, B (1996), pp.2679-2686.

(13) Sasoh, A., Onodera, O., Takayama, K., Kaneko, R. and Matsui, Y., Experimental Investigation on the Reduction of Railway Tunnel Sonic Boom, Trans. Jpn. Soc. Mech. Eng., (in Japanese), Vol.60, No.580, B (1994), pp.4112-4118.

(14) Maeda, T., Iida, M., Murata, K. and Fukuda, T., MicroPressure Waves Radiating from a Tunnel Portal and Their Mitigation, Proc. 29th Int. Congress Noise Control Eng., Paper IN2000/920 (2000).

(15) Ono, S., Okumura, Y., Fukuda, T. and Murata, K., Model Experiment of Train Nose Shape Optimization to Reduce Micro-Pressure Waves in Tunnels with En- 
trance Hoods, Preprint of J-RAIL'98, (in Japanese), (1998), pp.139-142.

(16) Yamauchi, N., Iida, M., Nakanishi, M. and Ono, A., Model Experiments on Tunnel Entrance Hoods for Reducing Micro-Pressure Wave, Preprint of Jpn. Soc. Mech. Eng., (in Japanese), No.98-37 (1998), pp.413414.

(17) Iida, M., Murata, K. and Fukuda, T., Estimation of the Necessary Length of a Tunnel Entrance Hood Entered into by Different Kinds of Trains, RTRI Report, (in Japanese), Vol.16, No.12 (2002), pp.35-40.

(18) Howe, M.S., Review of the Theory of the Compression Wave Generated When a High-Speed Train Enters a Tunnel, Proc. Inst. Mech. Eng., Part F: J. Rail and Rapid Transit, Vol.213 (1999), pp.89-104.

(19) Howe, M.S., Iida, M., Fukuda, T. and Maeda, T., Theoretical and Experimental Investigation of the Compression Wave Generated by a Train Entering a Tunnel with a Flared Portal, J. Fluid Mech., Vol.425 (2000), pp.111-132.

(20) Howe, M.S., Iida, M., Fukuda, T. and Maeda, T.,
Aeroacoustics of a Tunnel-Entrance Hood with a Rectangular Window, J. Fluid Mech., Vol.487 (2003), pp.211-243.

(21) Ogawa, T. and Fujii, K., Prediction of Wave Front of Compression Wave Generated by a Train Moving into a Tunnel with Steady Flow, Trans. Jpn. Soc. Mech. Eng., (in Japanese), Vol.61, No.586, B (1995), pp.2136-2142.

(22) Tanaka, Y., Iida, M. and Kikuchi, K., Method to Simulate Generation of Compression Wave inside a Tunnel at Train Entry with a Simple Geometry Model, Trans. Jpn. Soc. Mech. Eng., (in Japanese), Vol.69, No.683, B (2003), pp.1607-1614.

(23) Kikuchi, K., Maeda, T. and Yanagizawa, M., Numerical Simulation of the Phenomena due to the Passing by of Two Bodies Using the Unsteady Boundary Element Method, Int. J. Numerical Methods in Fluids, Vol.23 (1996), pp.445-454.

(24) Hara, T., Method of Measuring the Aerodynamic Drag of Trains, Bulletin of JSME, Vol.8, No.31 (1965), pp.390-396. 